\title{
Chronic obstructive pulmonary disease: hospital and intensive care unit outcomes in the Kingdom of Saudi Arabia
}

This article was published in the following Dove Press journal:

International Journal of COPD

17 December 2012

Number of times this article has been viewed

\author{
Abdulsalam M Alaithan' \\ Javed I Memon' \\ Rifat S Rehmani \\ Arif A Qureshi' \\ Abdul Salam ${ }^{3}$ \\ 'Department of Medicine, King \\ Abdulaziz Hospital, Al-Hasa, \\ Saudi Arabia; ${ }^{2}$ Department of \\ Emergency Medicine, King Abdulaziz \\ Hospital, Al-Hasa, Saudi Arabia; \\ ${ }^{3}$ King Abdullah International Medical \\ Research Center - Eastern Region \\ (KAIMRC-ER), Al-Hasa, Saudi Arabia
}

Correspondence:Abdulsalam Alaithan Consultant Pulmonary, Critical Care and Sleep Medicine, King Abdulaziz Hospital, Al-Hasa, Saudi Arabia

Tel +9663591000 ext 33445

Fax +9663591000 ext 33455

Email Alaithan@gmail.com
Background: There is little data surrounding the survival of patients with chronic obstructive pulmonary disease (COPD) who are admitted to the critical care unit with exacerbation of symptoms. We conducted a study to measure the in-hospital and intensive care unit (ICU) outcomes of patients admitted with COPD exacerbation, and identified the related prognostic factors.

Method: We performed a retrospective cohort study of patients who were admitted to the adult ICU between January 2006 and July 2011 for COPD exacerbation in King Abdulaziz National Guard Hospital, Al-Hasa, Saudi Arabia.

Results: During the study period, a total of 119 patients were admitted to the ICU with acute respiratory failure attributed to COPD exacerbation. The mean age was $72 \pm 13$ years, and $44(37 \%)$ were females. The main cause of respiratory failure was infection, which occurred in $102(86 \%)$ patients. Thirty-nine $(33 \%)$ of the admitted patients were mechanically ventilated, and the median duration was $2.6(1-42)$ days. The median lengths of the ICU and hospital stays were $3(1-40)$ and $9(2-43)$ days, respectively. The ICU mortality was $6 \%$, and hospital mortality was $11 \%$. Low Glasgow Coma Scale on admission, intubation, duration of mechanical ventilation, current smoking, tracheostomy, cardiopulmonary arrest, and the development of acute renal failure were associated with higher hospital mortality.

Conclusion: Early ICU and hospital mortality is low for COPD patients who have been admitted to the ICU with exacerbation. Low Glasgow Coma Scale scores on admission, intubation, prolonged use of mechanical ventilation, and the development of acute renal failure were identified as risk factors associated with increased hospital mortality.

Keywords: intensive care unit, chronic obstructive pulmonary disease, mortality rates, acute respiratory failure

\section{Introduction}

Chronic obstructive pulmonary disease (COPD) is a disease the incidence of which continues to increase. ${ }^{1}$ It is the leading cause of death worldwide, with a rising mortality rate, and, by the year 2020, it is expected that this will be the fifth leading condition among conditions with a high socioeconomic burden to society. ${ }^{2}$ The disease course is characterized by a progressive reduction in pulmonary function and recurrent exacerbations that may lead to acute respiratory failure, requiring intensive care unit (ICU) admissions for ventilation support, and the consumption of large ICU resources. The prognosis of these patients, particularly those who require invasive mechanical ventilation (IMV), is poor. The in-hospital mortality rate from COPD exacerbation is between $8 \%$ and $25 \%,,^{3-5}$ and the 1-year mortality rate after discharge from the ICU increases to between $35 \%$ and $48 \%$. ${ }^{5,6}$ Prognostic factors for in-hospital mortality and long-term 
outcomes have been identified to assist in clinical decision making and ICU management for the better utilization of medical resources. $3,4,7,8$

To the best of our knowledge, mortality due to COPD exacerbation in the ICU and its predictors has not yet been reported by the Kingdom of Saudi Arabia. Therefore, we present a retrospective cohort of COPD patients admitted to our ICU, their outcomes in terms of mortality rates, and predictors of hospital mortality.

\section{Methods}

The medical records of all patients admitted to the ICU in King Abdulaziz National Guard hospital in Al-Hasa, Saudi Arabia with a diagnosis of COPD during the period January 1, 2006 to July 31,2011 were actively reviewed. Admission with acute respiratory failure secondary to COPD exacerbation was also included. COPD was defined according to the Global Initiative for Obstructive Lung Disease, ${ }^{9}$ and it was identified from the patient's premorbid pulmonary function testing results; if this information was unavailable, data were gathered from the patient history with compatible physical examination findings, which are documented in each patient's chart. Exacerbation was defined by the presence of at least two of the three following symptoms: dyspnea, cough, and increasing of sputum purulence. Patients were excluded from the study if they had postoperative status, trauma, or if they were COPD patients admitted for diagnoses other than exacerbation.

The following data were extracted from the patient charts: demographic characteristics, comorbidities, APACHE II score, smoking history, exacerbation causes, Glasgow Coma Scale on admission, arterial blood gas upon admission and discharge, use of invasive and noninvasive mechanical ventilation, and the outcome results of each admission. The outcome variables include the following: ICU mortality, hospital mortality, duration of mechanical ventilation, tracheotomy, cardiopulmonary arrest, and the development of acute renal failure during hospital admission. We analyzed the first ICU admissions per patient hospitalization. The research proposal was approved by the King Abdullah International Medical Research Center (KAIMRC), Al-Hasa, Saudi Arabia.

Ventilation management during the ICU stay was initiated based on the judgment of the attending staff physician; however, noninvasive positive pressure ventilation (NIPPV) was used when applicable. All patients received standard treatment for COPD exacerbation, which includes bronchodilators, systemic corticosteroids, and appropriate antibiotics. Weaning from mechanical ventilators was performed according to the weaning protocol. ${ }^{10}$ Sedation, analgesia, and blood sugar management were provided as per their respective protocol in our ICU. ${ }^{11}$

\section{Statistical analysis}

Descriptive results were presented as the mean \pm standard deviation for all quantitative variables (eg, age) if normally distributed, and the median with minimum and maximum values was presented if the data were not normally distributed. Numbers (percentages) were reported for all qualitative variables (eg, sex). New binary variable status at discharge (alive versus dead) were created based on ICU or hospital mortality to compare all of the demographic and clinical characteristics between the two groups. Univariate analysis was performed using an independent samples $t$-test, or the Mann-Whitney U tests, if appropriate, for all quantitative variables. The Pearson chi-squared test, or the Fisher's exact test, if appropriate, for all qualitative variables was also performed to compare patients' mortality rates.

Multiple logistic regression models were built to identify the significant independent factors associated with survival. To build the model, a purposeful selection method was used to select a subset of covariates that were considered to be clinically important, adjusting for confounders and statistical significance. A purposeful selection of covariates begins with a multivariate model that contains all variables that are significant in the bivariate analysis at the $20 \%-25 \%$ level, as well as any other variables not selected with this criterion, but judged to be of clinical importance. We used $P$-values from the Wald tests of the individual coefficient to identify covariates that might be deleted from the model, and the $P$-values of the partial likelihood ratio test confirmed that the deleted covariates were not significant. Following the fitting of the reduced model, we assessed whether the removal of the covariate produced an "important" change (approximately 20\%) in the coefficient of the variables remaining in the model. The final model was assessed using the goodness-of-fit test to see if the model fit the data well. An adjusted odds ratio and a 95\% confidence interval (CI) were reported for each independent factor. Two-tailed $P$-values $<0.05$ were considered statistically significant. All statistical analyses were performed using SPSS (Statistical Package for the Social Sciences version 19.0, IBM Corporation, Armonk, NY).

\section{Results}

Overall, 178 COPD patients were admitted to the adult ICU over the study period, and 59 patients were excluded because they were not hospitalized based on COPD exacerbation. Thus, 119 patients were included in the study population.

The patients' characteristics and features are listed in Table 1 . The mean age was $72 \pm 13.3$ years; one-third of 
Table I Demographic and clinical characteristics of patients

\begin{tabular}{|c|c|}
\hline Factor & Results \\
\hline Total patients, $\mathrm{n}$ & 119 \\
\hline Age (years), mean \pm SD & $72 \pm 13$ \\
\hline Female, n (\%) & $44(37)$ \\
\hline APACHE II score, mean \pm SD & $17.75 \pm 6.3$ \\
\hline \multicolumn{2}{|l|}{ Comorbidity } \\
\hline Diabetes, n (\%) & $95(80)$ \\
\hline Hypertension, n (\%) & I0I (85) \\
\hline Coronary artery diseases, n (\%) & $52(44)$ \\
\hline Chronic renal failure, n (\%) & $21(18)$ \\
\hline Malignancy, n (\%) & $12(10)$ \\
\hline Anticoagulation, n (\%) & $33(28)$ \\
\hline Exsmoker, n (\%) & $38(32)$ \\
\hline \multicolumn{2}{|l|}{ Causes of exacerbation } \\
\hline Infection, n (\%) & $102(86)$ \\
\hline Noninfection, n (\%) & $17(14)$ \\
\hline \multicolumn{2}{|l|}{ Spirometry } \\
\hline $\mathrm{FEV}_{1}(\%)$, mean $\pm \mathrm{SD}$ & $44.89 \pm 16.2$ \\
\hline $\mathrm{FEV}_{\mathrm{I}} / \mathrm{FVC}(\%)$, mean $\pm \mathrm{SD}$ & $49.87 \pm 11.25$ \\
\hline \multicolumn{2}{|l|}{ Arterial blood gas on admission } \\
\hline $\mathrm{pH}$, mean $\pm \mathrm{SD}$ & $7.26 \pm 0.12$ \\
\hline $\mathrm{PCO}_{2}(\mathrm{mmHg})$, mean $\pm \mathrm{SD}$ & $65.71 \pm 20.78$ \\
\hline \multicolumn{2}{|l|}{ Conscious level on admission } \\
\hline Glasgow Coma Scale $\leq 10, \mathrm{n}(\%)$ & $19(16)$ \\
\hline \multicolumn{2}{|l|}{ Ventilatory management } \\
\hline Patients received IMV, n (\%) & $39(33)$ \\
\hline Duration of MV (days), median (range) & $2.5(I-42)$ \\
\hline Patients received NIPPV, n (\%) & $66(55)$ \\
\hline Patients intubated after failing NIPPV, $\mathrm{n}(\%)$ & $8(12)$ \\
\hline Patients received NIPPV after extubation, $\mathrm{n}(\%)$ & $19(16)$ \\
\hline
\end{tabular}

Abbreviations: APACHE II, Acute Physiology and Chronic Health Evaluation II; FEV, forced expiratory volume in first second; FVC, forced vital capacity; IMV, invasive mechanical ventilation; MV, mechanical ventilation; $n$, number; NIPPV, noninvasive positive pressure ventilation; $\mathrm{PCO}_{2}$, partial pressure of carbon dioxide.

participants were more than 65 years old, and the majority were male. The mean APACHE II score was $17.75 \pm 6.3$. Sixty-six patients $(55 \%)$ had two or more comorbidities, of which nearly half were attributable to type 2 diabetes and hypertension. A total of $86 \%$ of the patients had infective exacerbation of COPD. One-third of the patients received IMV for a median duration of 2.5 days. Sixty-six (55\%) patients received NIPPV as the initial treatment, but eight patients failed during its use and received IMV (Table 1).

The hospital mortality rate was $11 \%$, and seven of these cases $(6 \%)$ died in the ICU. The median ICU and hospital stays were 3 and 9 days, respectively. Nine percent of the patients received a tracheostomy, and $48 \%$ were discharged home with a home oxygen device supplement. Few patients had severe complications related to the severity of COPD exacerbation, such as cardiopulmonary arrest (3\%) and acute renal failure (5\%) (Table 2).

Patients who died were significantly more likely to have had lower Glasgow Coma Scale scores; they were also more
Table 2 The outcomes of COPD patients admitted to the ICU

\begin{tabular}{ll}
\hline Factor & Results \\
\hline ICU mortality, n (\%) & $7(6)$ \\
Hospital mortality, n (\%) & I $3(\mathrm{II})$ \\
ICU stay (days), median (range) & $3(\mathrm{I}-40)$ \\
Hospital stay (days), median (range) & $9(2-43)$ \\
Post-ICU hospital stay (days), median (range) & $4(\mathrm{I}-40)$ \\
Tracheotomy, n (\%) & $\mathrm{II}(9)$ \\
Cardiopulmonary arrest, n (\%) & $3(3)$ \\
Acute renal failure, $\mathrm{n}(\%)$ & $6(5)$ \\
Home $\mathrm{O}_{2}$ device on discharge, $\mathrm{n}(\%)$ & $57(48)$ \\
\hline
\end{tabular}

Abbreviations: COPD, chronic obstructive pulmonary disease; ICU, Intensive Care Unit; n, number.

likely to have developed acute renal failure, had a tracheostomy, experienced cardiopulmonary arrest, and to have been smokers at the time of the study. The duration of IMV was significantly higher among those who died versus those who were alive, with an associated $P$-value of 0.002 (Table 3).

The multiple logistic regression model identified that Glasgow Coma Scale scores, the development of acute renal failure, and being a smoker were significant independent prognostic factors associated with survival (Table 4). Patients aged 65 or older were included in the model. The results of the regression analysis showed that age $>65$ (odds ratio [OR] $=0.064,95 \%$ CI: 0.003-1.44), Glasgow Coma Scale score $\leq 10(\mathrm{OR}=1.24$, 95\% CI: 1.042-1.76), the development of acute renal failure (OR $=0.026,95 \% \mathrm{CI}: 0.002-0.308)$, and current smoking status $(\mathrm{OR}=0.062,95 \% \mathrm{CI}: 0.01-0.389)$ were the most significant contributing factors leading to patient mortality.

\section{Discussion}

In our study, we found that the hospital mortality rates of COPD patients admitted with respiratory failure due to COPD exacerbation was $11 \%$ and ICU mortality rate

Table 3 Predictors of hospital mortality

\begin{tabular}{llll}
\hline Factor & Nonsurvival & Survival & $P$-value \\
\hline $\begin{array}{l}\text { Duration of MV (days), median } \\
\text { (range) }\end{array}$ & $6(I-42)$ & $2(I-25)$ & $0.002^{\psi}$ \\
Glasgow Coma Scale $\leq 10$ & $6(46)$ & $13(I I)$ & 0.006 \\
Intubation & $9(69)$ & $3 I(27)$ & 0.010 \\
Tracheotomy & $4(3 I)$ & $7(6)$ & 0.019 \\
CPR & $2(I 5)$ & $I(I)$ & 0.031 \\
ARF & $4(3 I)$ & $2(2)$ & 0.001 \\
Currently smoking & $4(3 I)$ & $8(7)$ & 0.026 \\
\hline
\end{tabular}

Notes: Results are expressed as number (percentage) unless otherwise specified. ${ }^{\Psi} P$-value has been calculated using the Mann-Whitney $U$ tests; ${ }^{\circledR} P$-value has been calculated using the Pearson Chi-squared test or the Fisher's exact test whenever appropriate, unless otherwise specified.

Abbreviations: MV, mechanical ventilation; CPR, cardiopulmonary resuscitation; ARF, acute renal failure. 
Table 4 The results obtained from the multiple logistic regression analysis, indicating prognostic factors for hospital survival $(n=106$ alive versus 13 in-hospital deaths)

\begin{tabular}{|c|c|c|c|}
\hline Factor & $\begin{array}{l}\text { Adjusted } \\
\text { odds ratio }\end{array}$ & $\begin{array}{l}95 \% \mathrm{Cl} \text { for } \\
\text { adjusted odds ratio }\end{array}$ & $P$-value \\
\hline Age $>65$ & 0.064 & $0.003-1.44$ & 0.084 \\
\hline GCS & 1.24 & $1.042-1.476$ & 0.015 \\
\hline ARF & 0.026 & $0.002-0.308$ & 0.004 \\
\hline Current smoker & 0.062 & $0.010-0.389$ & 0.003 \\
\hline
\end{tabular}

Abbreviations: GCS, Glasgow Coma Scale; ARF, acute renal failure.

was $6 \%$. The overall hospital mortality was lower than mortality rates reported in other studies. In Spain, Raurich et $\mathrm{al}^{4}$ reported an in-hospital mortality of $26 \%$ among COPD patients who were admitted to the ICU and received IMV due to COPD exacerbation. This was attributed to prolonged IMV use, as well as more days spent in ICU and hospitalization. Ai-Ping et $\mathrm{al}^{3}$ reported a $24 \%$ in-hospital mortality rate during ICU admission in 2004. Ninety percent of their cohort received mechanical intubations, which explains the magnitude of these higher mortality rates. This could be explained by several reasons. First, the early use of noninvasive mechanical ventilation was associated with a high success rate $(84 \%)$. Moreover, noninvasive MV has been shown to improve patient outcomes and avoid endotracheal intubation. ${ }^{8,12,13}$ Second, our population sample had a lower APACHE II score compared to others. Third, fewer patients required mechanical ventilation and prolonged ICU and hospital stays, which other studies have demonstrated to increase in-hospital mortality. ${ }^{13,14}$

Multiple risk factors were found to be associated with early ICU mortality. A low Glasgow Coma Scale score equal to or less than ten, intubation, tracheostomy, cardiopulmonary arrest, the development of acute renal failure during ICU admission, and status as a current smoker were found to be associated with increased hospital mortality; this has been described in the literature. Afessa et $\mathrm{al}^{7}$ found that previous intubation, age, duration of hospital stay, and APACHE II score were significantly correlated with higher mortality in COPD patients that were admitted to the ICU with exacerbation. Interestingly, our study identified the presence of acute renal failure as a risk factor for increasing mortality, which was not known before. This may elucidate the importance of intravenous fluid and use of diuretics in critical care.

Of particular interest is that $37 \%$ of the patients were females who had never smoked. The development of COPD among this population has been attributed to the extensive use of incense burners, especially among Saudi women, who use them for traditional perfumes. ${ }^{14}$ Furthermore, the problem of the misdiagnosis of asthma and COPD is common, considering that one-fifth of elderly asthma patients are misdiagnosed as having COPD. ${ }^{15}$

Our study has several limitations related to both its design and it being a single-center study. We had no information related to the functional status of the patients and their compliance with treatment. Although our ICU has a standardized protocol for weaning IMV, alternative medical plans and NIPPV treatment were most likely determined by the individual physician's preference, which may have affected the outcomes.

In conclusion, COPD patients admitted to the ICU due to COPD exacerbation have a good chance of surviving until hospital discharge. Multiple independent risk factors, including the development of acute renal failure, low consciousness level, and a patient's status of being a current smoker significantly increase in-hospital mortality. Further study is required to identify the long-term survival rates of this population.

\section{Acknowledgments}

The authors would like to acknowledge Dr Alaa Ghabashi, Dr Syed Tabish Zaydi, and Mr Mohammed Elarab for their help and support, without which the study would not have been possible.

\section{Disclosure}

The authors report no conflicts of interest in this work.

\section{References}

1. Mannino DM, Watt G, Hole D, et al. The natural history of chronic obstructive pulmonary disease. Eur Respir J. 2006;27(3):627-643.

2. Hurd S. The impact of COPD on lung health worldwide: epidemiology and incidence. Chest. 2000;117(Suppl 2):1S-4S.

3. Ai-Ping C, Lee KH, Lim TK. In-hospital and 5-year mortality of patients treated in the ICU for acute exacerbation of COPD: a retrospective study. Chest. 2005;128(2):518-524.

4. Raurich JM, Pérez J, Ibáñez J, Roig S, Batle S. In-hospital and 2-year survival of patients treated with mechanical ventilation for acute exacerbation of COPD. Arch Bronconeumol. 2004;40(7):295-300.

5. Groenewegen KH, Schols AM, Wouters EF. Mortality and mortalityrelated factors after hospitalization for acute exacerbation of COPD. Chest. 2003;124(2):459-467.

6. Breen D, Churches T, Hawker F, Torzillo PJ. Acute respiratory failure secondary to chronic obstructive pulmonary disease treated in the intensive care unit: a long term follow up study. Thorax. 2002;57(1): 29-33.

7. Afessa B, Morales IJ, Scanlon PD, Peters SG. Prognostic factors, clinical course, and hospital outcome of patients with chronic obstructive pulmonary disease admitted to an intensive care unit for acute respiratory failure. Crit Care Med. 2002;30(7):1610-1615.

8. Nevins ML, Epstein SK. Predictors of outcome for patients with COPD requiring invasive mechanical ventilation. Chest. 2001;119(6): 1840-1849. 
9. Celli BR, MacNee W; for ATS/ERS Task Force. Standards for the diagnosis and treatment of patients with COPD: a summary of the ATS/ERS position paper. Eur Respir J. 2004;23(6):932-946.

10. Iqbal M, Rehmani R, Venter J, Alaithan AM. Quality assurance in an adult intensive care unit, Eastern region, Saudi Arabia. Saudi Med J. 2007;28(3):408-411.

11. Iqbal M, Al-Aithan AM, Rehmani R, Eledrisi M. Initial experience with an intensive care hyperglycemia protocol in a Saudi Arabian intensive care unit. Saudi Med J. 2006;27(4):492-496.

12. Plant PK, Owen JL, Elliott MW. Early use of non-invasive ventilation for acute exacerbations of chronic obstructive pulmonary disease on general respiratory wards: a multicentre randomised controlled trial. Lancet. 2000;355(9219):1931-1935.
13. Chandra D, Stamm JA, Taylor B, et al. Outcomes of noninvasive ventilation for acute exacerbations of chronic obstructive pulmonary disease in the United States, 1998-2008. Am J Respir Crit Care Med. 2011;185(2):152-159.

14. Døssing M, Khan J, al-Rabiah F. Risk factors for chronic obstructive lung disease in Saudi Arabia. Respir Med. 1994;88(7):519-522.

15. Al-Kassimi FA, Alhamad EH. Re: prevalence of chronic obstructive pulmonary disease among smokers attending primary healthcare clinics in Saudi Arabia. Ann Saudi Med. 2011;31(4):433-434; author reply 434.

International Journal of COPD

\section{Publish your work in this journal}

The International Journal of COPD is an international, peer-reviewed journal of therapeutics and pharmacology focusing on concise rapid reporting of clinical studies and reviews in COPD. Special focus is given to the pathophysiological processes underlying the disease, intervention programs, patient focused education, and self management protocols.

\section{Dovepress}

This journal is indexed on PubMed Central, MedLine and CAS. The manuscript management system is completely online and includes a very quick and fair peer-review system, which is all easy to use. Visit http://www.dovepress.com/testimonials.php to read real quotes from published authors.

Submit your manuscript here: http://www.dovepress.com/international-journal-of-copd-journal 